\title{
Generation of OAM Radio Waves with Three Polarizations Using Circular Horn Antenna Array
}

\author{
Xudong Bai, Ronghong Jin, Liang Liu, Junping Geng, and Xianling Liang \\ Department of Electronics Engineering, Shanghai Jiao Tong University, Shanghai 200240, China \\ Correspondence should be addressed to Xianling Liang; liangxl@sjtu.edu.cn
}

Received 29 August 2014; Revised 28 October 2014; Accepted 28 October 2014

Academic Editor: Giacomo Oliveri

Copyright ( 2015 Xudong Bai et al. This is an open access article distributed under the Creative Commons Attribution License, which permits unrestricted use, distribution, and reproduction in any medium, provided the original work is properly cited.

\begin{abstract}
This paper provides an effective solution of generating OAM-carrying radio beams with all three polarizations: the linear, the lefthand circular, and the right-hand circular polarizations. Through reasonable configuration of phased antenna array using elements with three polarizations, the OAM radio waves with three polarizations for different states can be generated. The vectors of electric fields with different OAM states for all three polarizations are presented and analyzed in detail. The superposition of two coaxial OAM states is also carried out, and the general conclusion is provided.
\end{abstract}

\section{Introduction}

Since the first radio signal was transmitted and received by Guglielmo Marconi in December 1895, the wireless communication has gone deep into all aspects of human life [1]. Today most of the information of human life is transmitted through wireless channels and the fast-growing mobile devices have led to congestion in the precious and finite radio frequency resource even after the application of many techniques which can increase the capacity of communication systems, such as orthogonal frequency-division multiplexing (OFDM) [2]. Therefore, it is very significant to develop some new techniques which can greatly increase the spectral efficiency. The orbital angular momentum (OAM) may be one good choice to utilize the electromagnetic spectrum better.

An electromagnetic wave radiates energy as well as angular momentum (AM) [3]. The angular momentum is composed of spin angular momentum (SAM) describing the polarization state and orbital angular momentum (OAM) describing the helical phase structures. Since SAM has been widely used starting around 1935 when it was experimentally demonstrated by Beth [4], OAM is not fascinating until Allen et al. investigated in 1992 that beams with helical wave fronts comprise an azimuthal phase term $\exp (i \ell \theta)$ and have an OAM of $\ell \hbar$ per photon (where $\ell$ is topological charge and $\theta$ is the azimuthal angle) [5]. At first, the applications of OAM have mostly been within the optical regime, and, by introducing OAM, the capacity of optical communication systems is largely extended $[6,7]$. It was not found until very recently that the photon OAM can be used in the low frequency radio domain and is not restricted to the optical frequency range [8]. From that moment on, many optical methods were introduced to give rise to OAM-carrying radio beams $[9,10]$, since the generating of OAM is one of the most important issues. However, all these methods can generate only a fixed-mode OAM radio beam; so, in [11, 12], the circular antenna array is introduced to generate OAM waves with different topological charges. In [11], simple dipole antenna is adopted as array element, and the elements are fed by the same signal, but with a successive phase delay, while in [12], Vivaldi antenna is used as array element, and Vivaldi antenna elements connect sequentially and fold into a hollow cylinder to generate OAM, but the simulation results are quite different from the traditional states.

In this document, we provide an effective solution of generating OAM-carrying radio beams with all three polarizations, including the linear polarization, the left-hand circular polarization (LHCP), and the right-hand circular polarization (RHCP), using phased circular horn antenna array. Through the reasonable configuration of the antenna array using elements with three polarizations, the OAM radio 

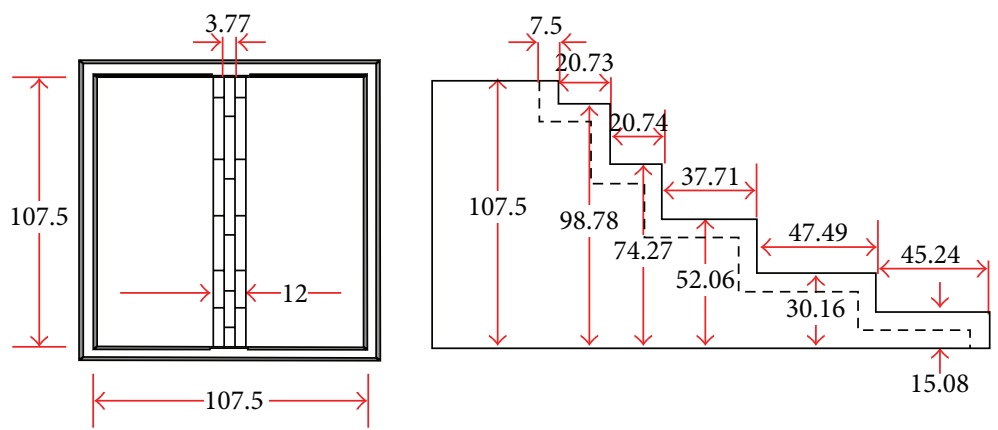

FIGURE 1: Geometry of three-port stepped-septum polarizer (item: $\mathrm{mm}$ ).

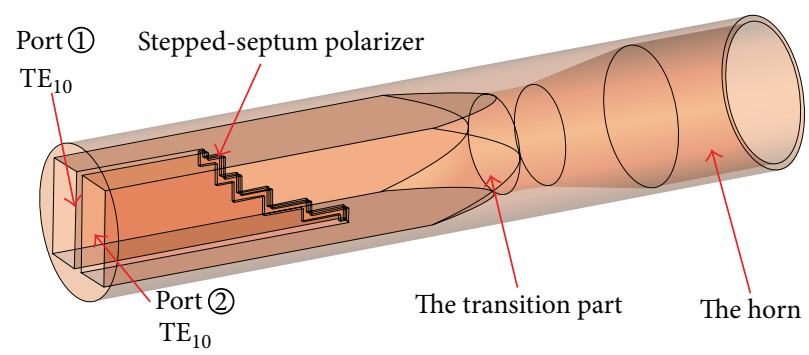

FIgURE 2: The holistic configuration of the horn antenna.

waves with all three polarizations for different states can be generated. When combining both the polarization states and OAM states, communication systems would have tremendous increases in capacity and spectral efficiency. The radiation patterns as well as electric fields of all three polarizations for different OAM states are analyzed in detail, and the simulation results are quite consistent with the traditional optical states. All of the research work was carried out with computer simulation technology (CST) Microwave Studio software based on the finite integration technique (FIT) method.

\section{Antenna Element}

The key component of the antenna element is a threeport stepped-septum polarizer which can excite high-purity LHCP and RHCP, as well as the linear polarization [13]. The input of the polarizer is composed of two identical rectangular waveguide ports while the output is a single square waveguide. When only one port is excited, the LHCP or RHCP is produced; while both input ports are synchronously excited with a proper phase, the linear polarization is obtained, which would be tilted at any angle with respect to the horizontal or vertical aperture direction. The configuration of the three-port stepped-septum polarizer is shown in Figure 1. The stepped-septum has six steps, and a groove is made on it to increase the isolation of the two ports. Figure 2 gives the holistic configuration of the horn antenna element, which consists of three parts: the three-port stepped-septum polarizer, the square-to-circular wave-guide transition, and the horn.

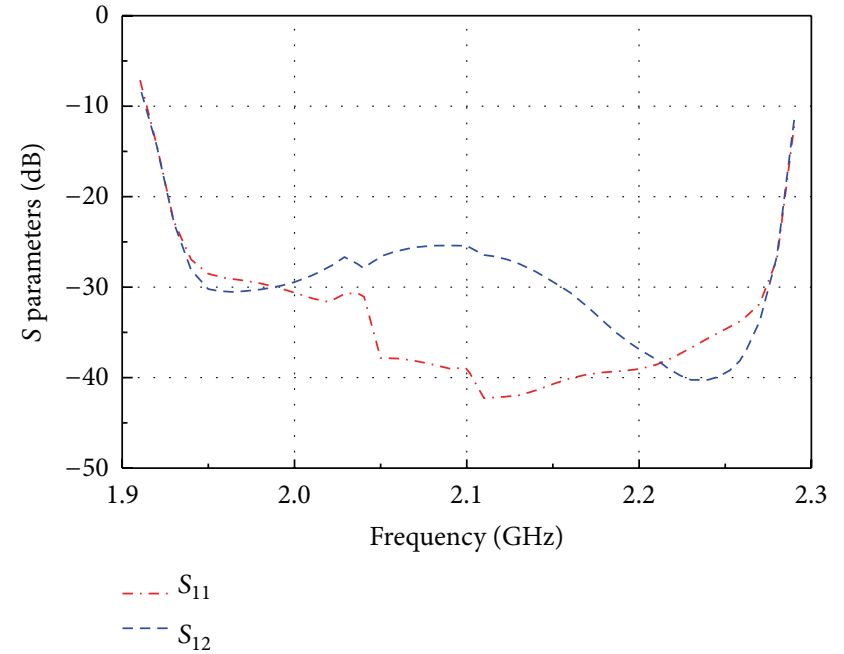

Figure 3: Simulated S-parameters of the horn antenna.

The simulated $S$-parameters of the horn antenna are shown in Figure 3; the return loss is below $-20 \mathrm{~dB}$ from 1.93 to $2.28 \mathrm{GHz}$ and the isolation between the two ports is more than $25 \mathrm{~dB}$ in the corresponding frequency band. The gain of the horn antenna is larger than $10.9 \mathrm{dBi}$ with a good directionality at $2.2 \mathrm{GHz}$ as shown in Figure 4 and the simulated axial ratio (AR) is below $2 \mathrm{~dB}$ when the theta varies from $-58^{\circ}$ to $59^{\circ}$. The simulated cross polarization discrimination of LHCP and RHCP is more than $30 \mathrm{~dB}$, as shown in Figure 4. 


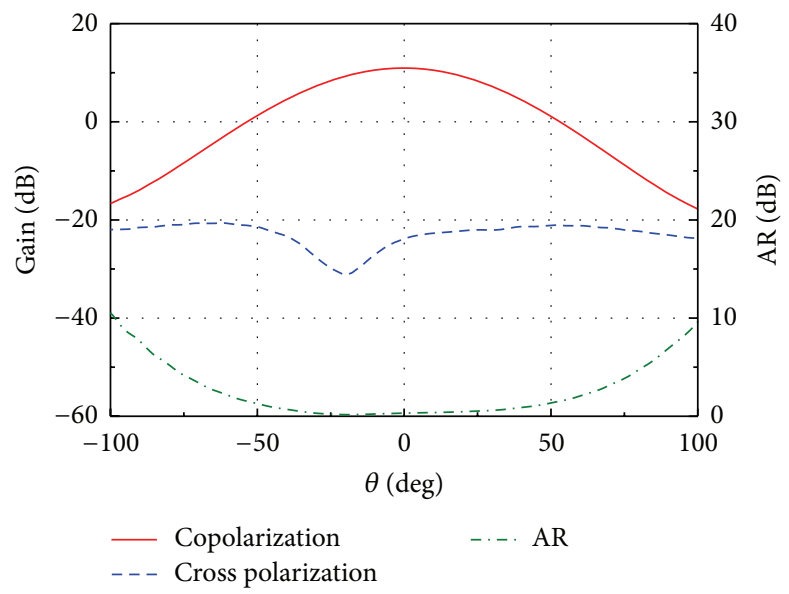

FIGURE 4: Simulated radiation patterns and AR of the horn antenna (2.2 GHz).

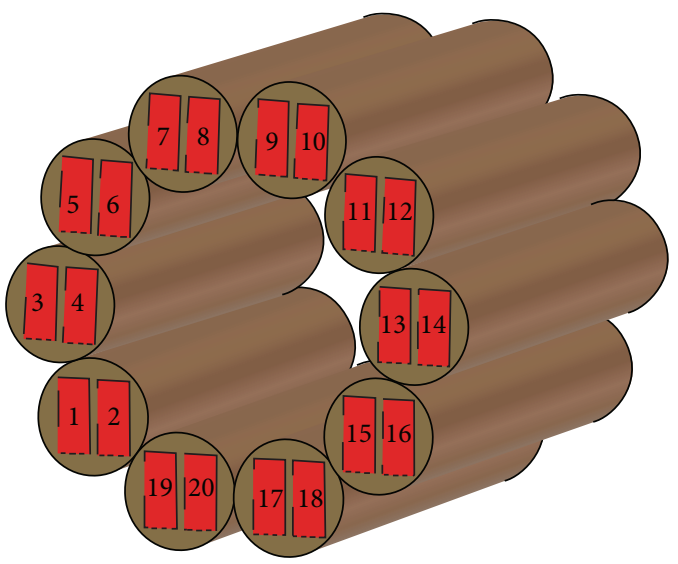

(a)

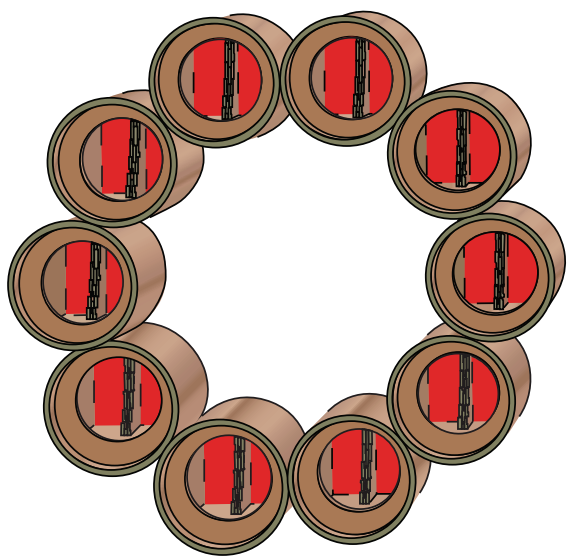

(b)

FIGURE 5: Geometry of the horn antenna array: (a) Back view. (b) Front view.

\section{Array Structure and Mechanism}

3.1. Array Structure. The configuration of the OAMgenerating array is shown in Figure 5. In our present work, $N=10$ horn antennas are equidistantly spaced along the perimeter of a circle with a diameter of $D=4 \lambda$; it is important to note that all elements should be oriented along the same direction with no rotation. To get helical wave fronts, the array should be fed with the uniform amplitude but with a successive phase difference for the successive elements, which is $\Delta \phi=2 \pi \ell / N=\ell * 36^{\circ}$ (where $\ell$ is topological charge). By changing the phase difference at the steps of $0^{\circ}, \pm 36^{\circ}, \pm 72^{\circ}, \pm 108^{\circ}$, and $\pm 144^{\circ}$, the OAM radio beams of $\ell=0, \pm 1, \pm 2, \pm 3$, and \pm 4 can be generated. Through simply choosing the excited ports and the right phase difference, perfect OAM-carrying radio beams with all three polarizations for different OAM states can be obtained. By encoding information as OAM states of the beam, the capacity of wireless communication systems would be greatly improved, and the spectral efficiency will be doubled when the polarization states are further introduced.

3.2. Radiation Patterns. Figure 6 gives the radiation patterns of the OAM-carrying radio beams with all possible states at the frequency of $2.2 \mathrm{GHz}$; correspondingly, the intensity distributions of Laguerre-Gaussian beams with different OAM states are shown in Figure 7. As you can see, the radiation patterns of the antenna array are quite consistent with the traditional optical states for OAM states $\ell=0, \pm 1, \pm 2$, and \pm 3 , while that for OAM state $\ell= \pm 4$ is slightly distorted. This is because the largest OAM mode that the array can generate is strictly restricted by the number of the elements, which is $|\ell|<N / 2$ (where $N$ is the number of array elements) [8-12]. It needs to be stressed that the radiation patterns of LHCP and RHCP as well as linear polarizations are all the same for the identical OAM state.

The spatial extent of the null region of the radio beams widens as the absolute value of the topological charge $\ell$ 


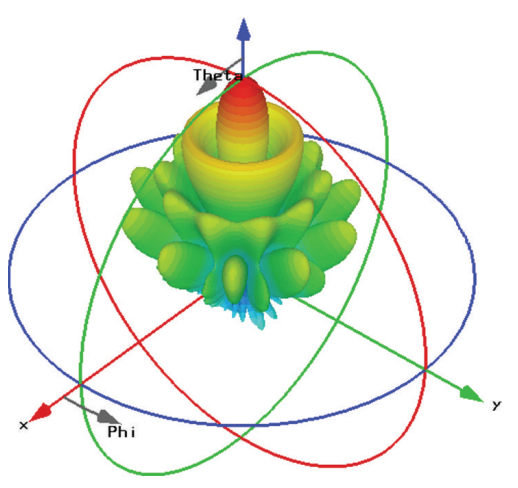

(a)

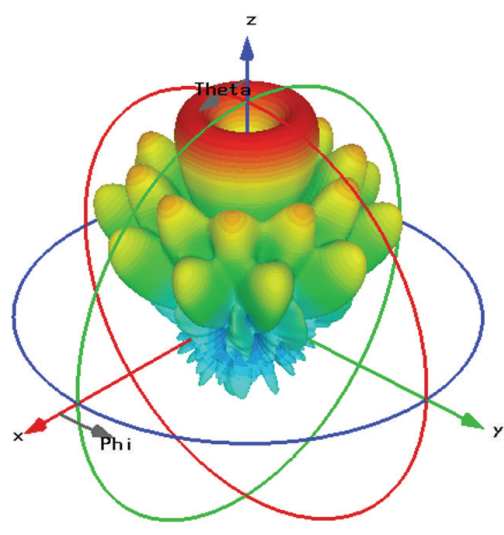

(c)

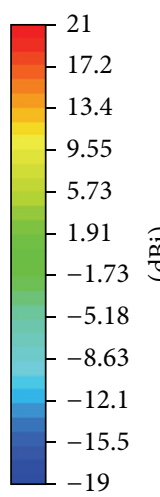

19
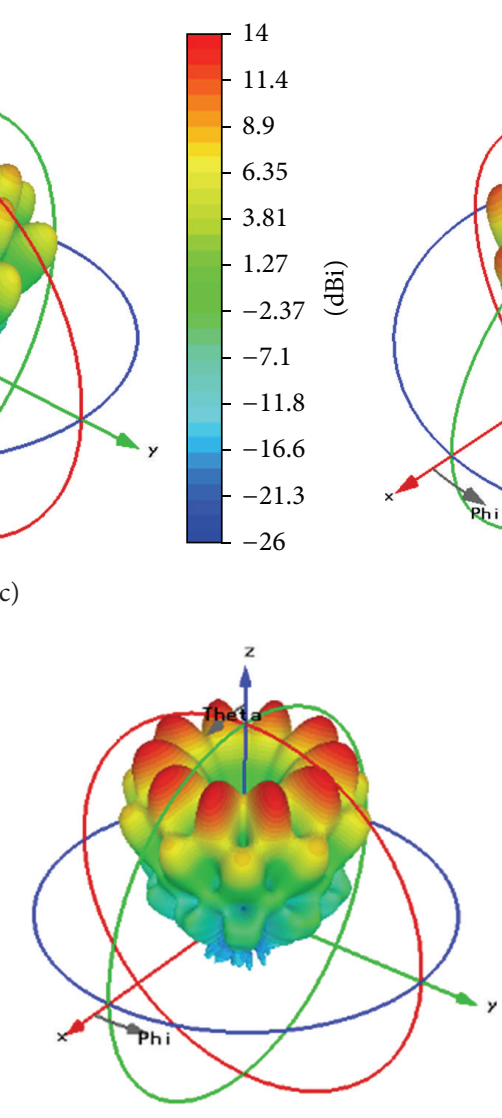

(e)

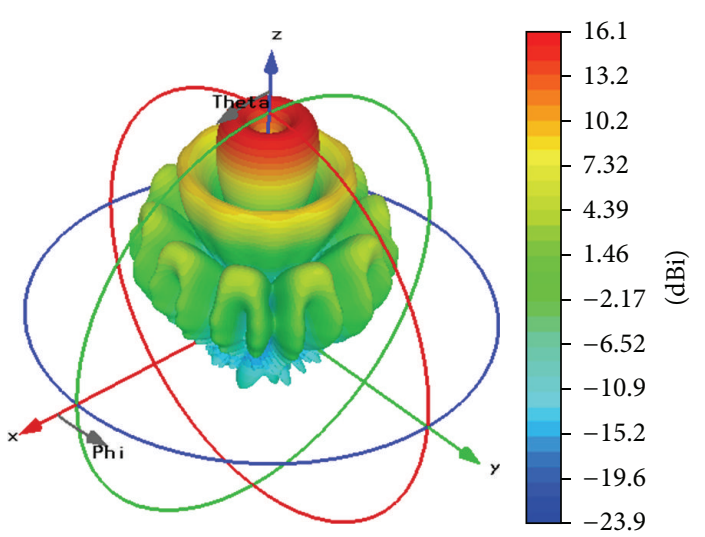

(b)

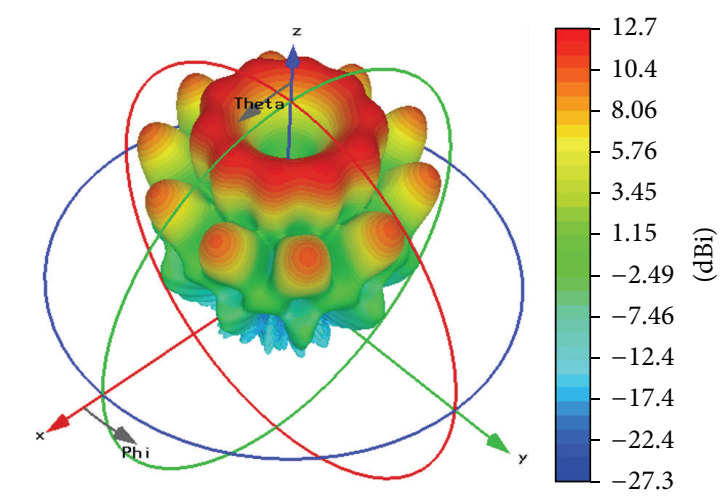

(d)

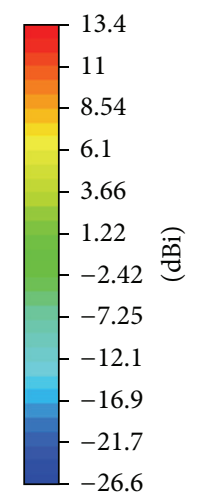

FIgURE 6: Radiation patterns with different OAM states at $2.2 \mathrm{GHz}$ : (a) $\ell=0$, (b) $\ell= \pm 1$, (c) $\ell= \pm 2$, (d) $\ell= \pm 3$, and (e) $\ell= \pm 4$.

increases, as is more clearly shown in Figure 8. In other words, the direction angle of the maximum gain becomes wider as the absolute value of the topological charge increases, which means that the receiving antennas for different OAM states should be placed at different places for the strong beam intensity. The relationship between the direction angle $\theta$ of the maximum gain and the topological charge $\ell$ is derived as follows:

$$
\frac{2 \pi}{\lambda} D \sin \theta=|\ell| \pi
$$

where $D$ is the diameter of the circular array.
From (1), we know there is a lower limit of the diameter of the circular array, which is $D>\lambda / 2$; for a certain topological charge $\ell$, the diameter $D$ of the circular array should be enlarged to minimize the area of the null region. Figure 9 shows the effect of diameter $D$ on the radiation patterns of mode $\ell=+3$. As the diameter of the array increases, the maximum gain increases while its direction angle decreases. The side lobes of the beam also increase when the array diameter is enlarged, but they are at much wider angles than the main lobe. This phenomenon is also clearly shown in Table 1. 


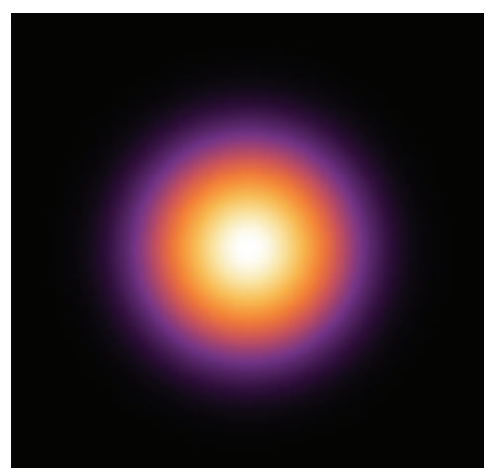

(a)

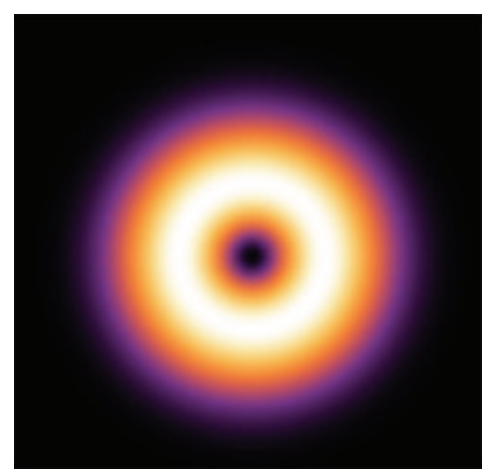

(b)

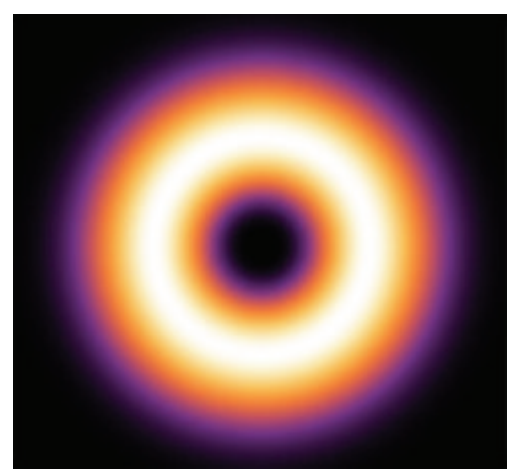

(c)

FIGURE 7: The intensity distributions of Laguerre-Gaussian beams with different OAM states: (a) $\ell=0$, (b) $\ell= \pm 1$, and (c) $\ell= \pm 2$ (from Wikipedia).

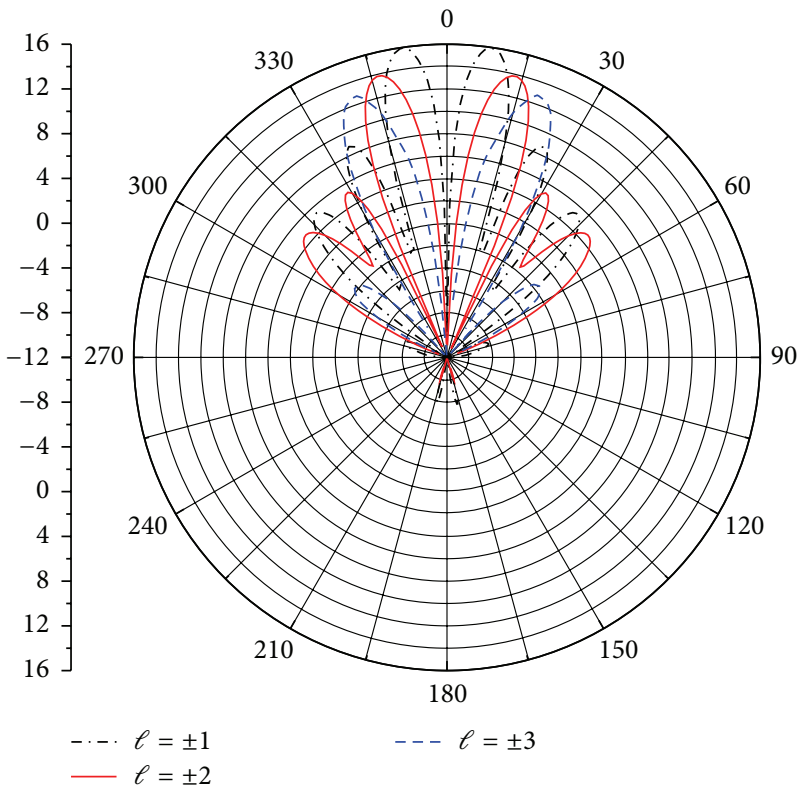

FIGURE 8: Radiation patterns with different OAM states in E-plane.

TABLE 1: Variation of the angle $\theta_{\text {main }}$ of the maximum gain $G_{\max }$ and angle $\theta_{\text {slob }}$ of the 1st side lobe with the array diameter $D$ for mode $\ell=+3$.

\begin{tabular}{lcccc}
\hline$D$ & $\theta_{\text {main }}$ & $G_{\max }[\mathrm{dB}]$ & $\theta_{\text {slob }}$ & $G_{1 \text { st sidelobe }}[\mathrm{dB}]$ \\
\hline $4 \lambda$ & $19^{\circ}$ & 12.4 & $55^{\circ}$ & -1.7 \\
$5 \lambda$ & $16^{\circ}$ & 12.9 & $41^{\circ}$ & 2.4 \\
$6 \lambda$ & $13^{\circ}$ & 13.4 & $32^{\circ}$ & 3.9 \\
\hline
\end{tabular}

3.3. Electric Fields. Figure 10 gives the vectors of the electric fields with different OAM states for the linear polarization. The focus area, where the vectors are plotted, is intersected at $20 \lambda$ from the transmitting array. The size of the concerned area is $16 \lambda \times 16 \lambda$. It is observed that the OAM states of $\pm 1, \pm 2$, and \pm 3 are distinguished easily when calculating the number of helical wave fronts in the clockwise or anticlockwise

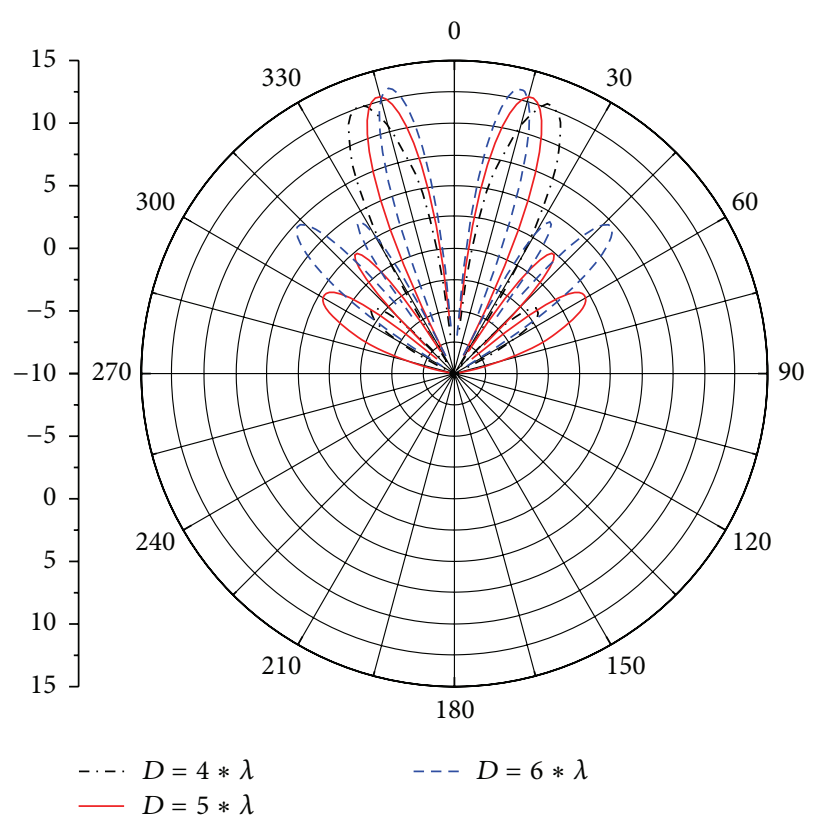

FIGURE 9: Radiation patterns of mode $\ell=+3$ with different array diameter $D$.

direction. A change in direction of the arrows from right to left and back to right again corresponds to a change in phase of $2 \pi$. When looking in the projection direction, the peak value spot of the electric field with negative $\ell$ value has a clockwise rotation, while that with the positive $\ell$ value would have an anticlockwise rotation.

The vectors of the electric fields with OAM state $\ell=+1$ for the LHCP are shown in Figure 11. It is observed that the electric fields for the circular polarization also reveal helical phase structures, and the peak value spot of the electric field has a clockwise or anticlockwise rotation around axis; for a fixed point, the electric field vectors conduct periodic rotation, which is a key feature of the circular polarization. 


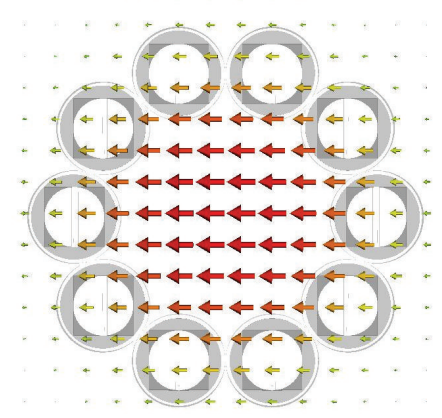

(a)

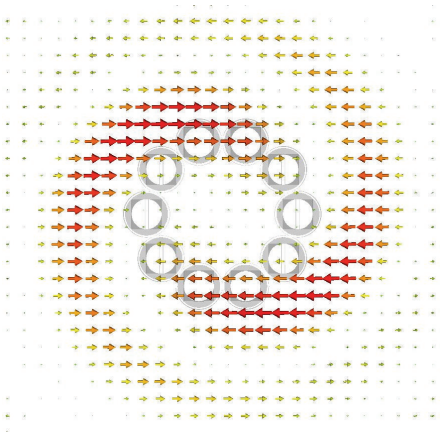

(b)

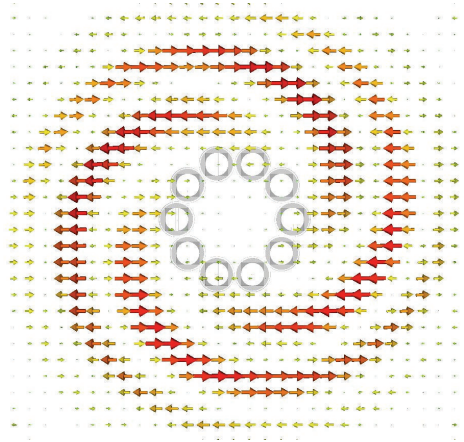

(d)

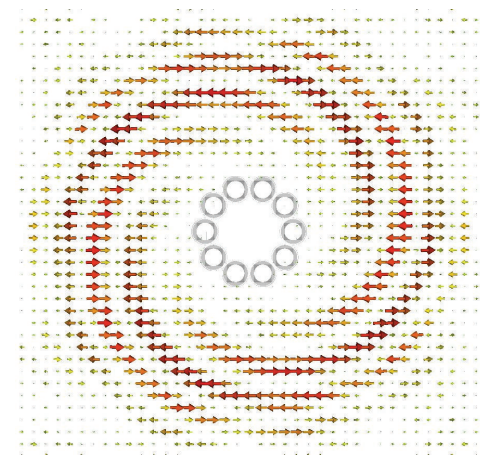

(f)

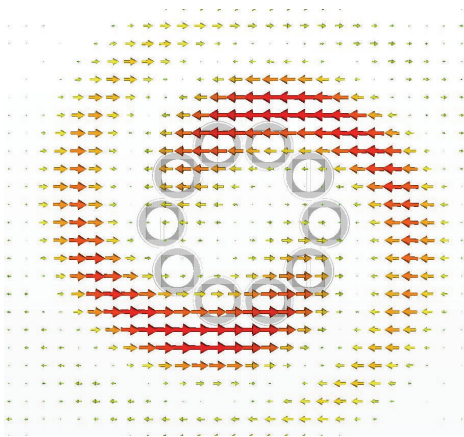

(c)

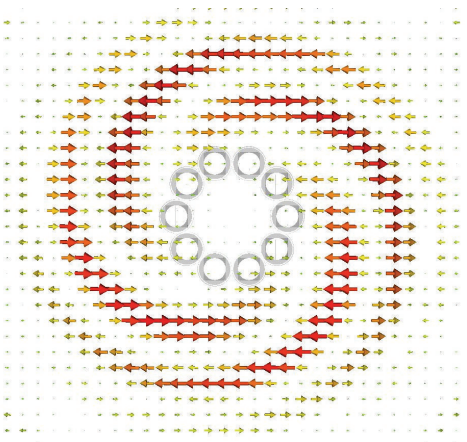

(e)

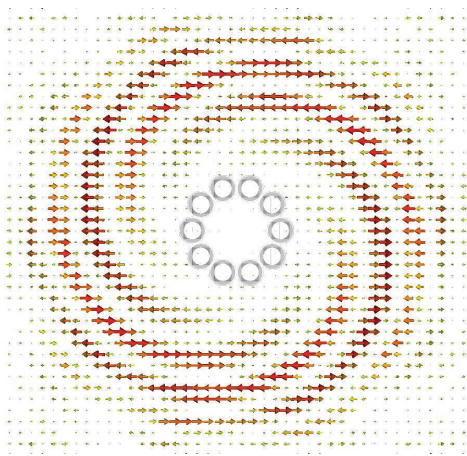

$(\mathrm{g})$

FIGURE 10: The vectors of electric field for linear polarization with different OAM states. (a) $\ell=0$, (b) $\ell=+1$, (c) $\ell=-1$, (d) $\ell=+2$, (e) $\ell=-2$, (f) $\ell=+3$, and (g) $\ell=-3$. 


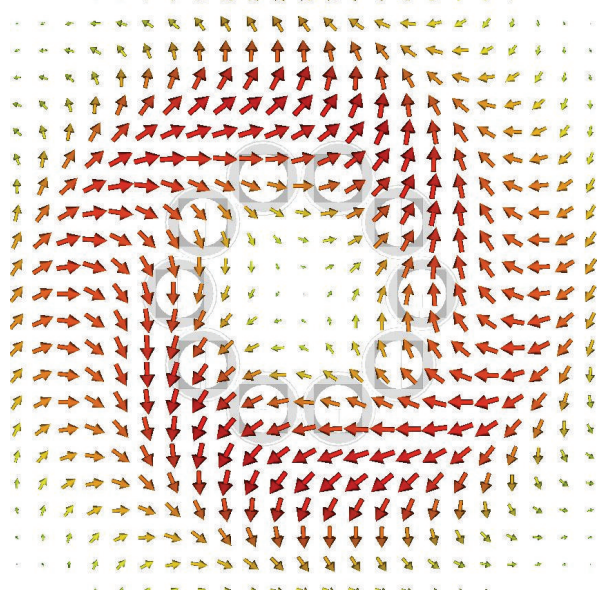

(a)

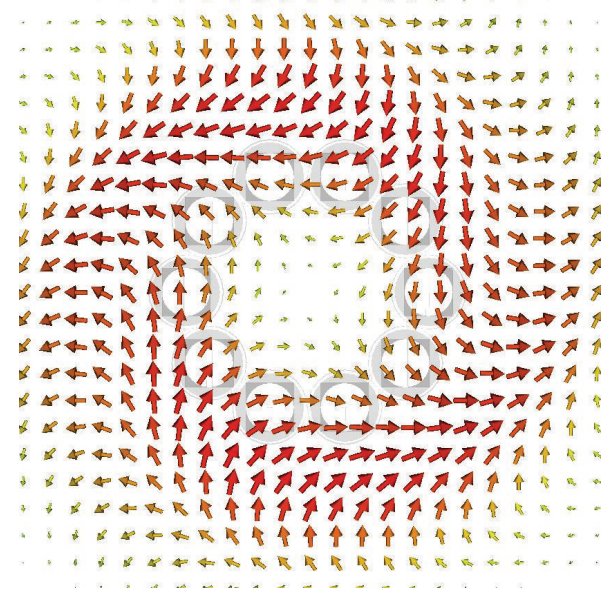

(c)

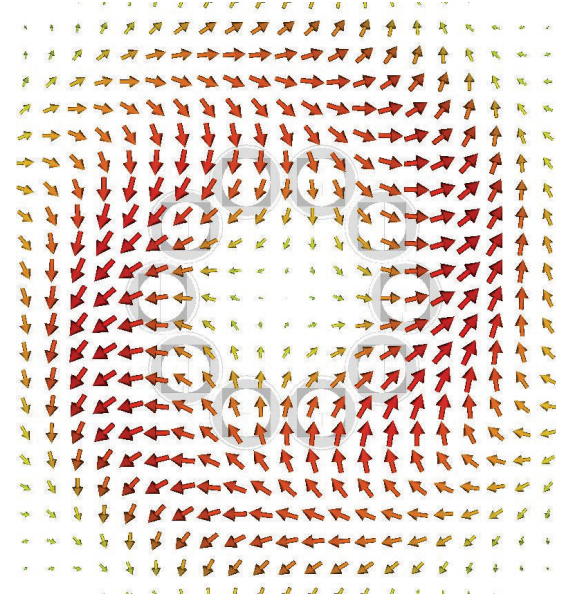

(b)

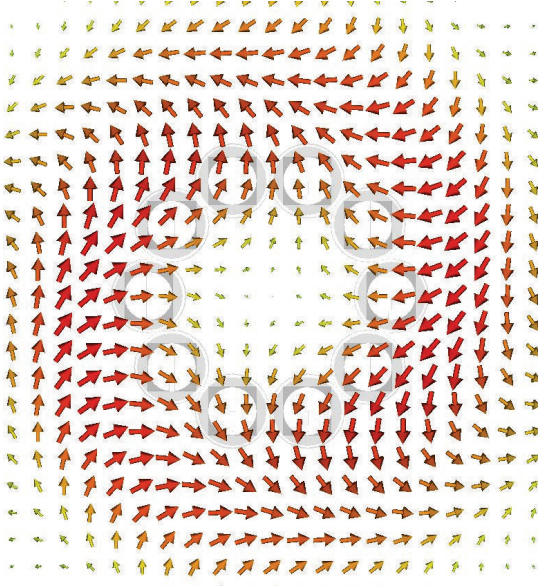

(d)

Figure 11: Electric field vectors with state $\ell=+1$ for left circular polarization in one period: (a) phase $=0^{\circ},(\mathrm{b})$ phase $=90^{\circ},(\mathrm{c})$ phase $=180^{\circ}$, and $(\mathrm{d})$ phase $=270^{\circ}$.

3.4. Helical Phase Map of OAM States. The phase maps of the OAM radio beams with topological charge $\ell=+1,+2$, and +3 for the liner and right circular polarizations are plotted in Figure 12, respectively. The focus area, where the phase maps are plotted, is also intersected at $20 \lambda$ from the transmitting array. Note that characteristic helical phase fronts of OAM radio beams are completely revealed. Though there is quite a great difference in electric field vectors between the linear and the circular polarizations, the phase maps of two polarizations show little difference, and the change in color from blue to green, yellow, and red and back to blue again corresponds to a change in phase of $2 \pi$.

3.5. AR for OAM. The AR of OAM states $\ell=-1$ for the RHC and $\ell=+3$ for the LHCP are plotted in Figures 13 and 14 , respectively, in which all demonstrate good circular polarizations for the main beams. Since the cross polarization discrimination of the LHCP and RHCP is over $30 \mathrm{~dB}$ for the horn antennas as shown in Figure 4, by further introducing the two circular polarization states, the spectral efficiency will be doubled.

In addition, characteristic on-axis phase singularities of OAM are also revealed on the AR-plots, and the spatial extent of the on-axis singular-phase null region widens as the absolute value of the topological charge $\ell$ increases, which are in correspondence with the phenomenon of the radiation patterns for OAM shown in Figure 8.

3.6. Superposition of OAM States. Since each of the OAM eigenstates with integral topological charges is orthogonal and independent of the other, a superimposed noninteger OAM state $\alpha \in R$ can then be decomposed into a Fourier series superposition of orthogonal integral eigenstates [14]; that is,

$$
\exp (i \alpha \varphi)=\frac{\exp (i \pi \alpha) \sin (\pi \alpha)}{\pi} \sum_{\ell=-\infty}^{+\infty} \frac{\exp (i \ell \varphi)}{\alpha-\ell}, \quad \ell \in Z .
$$




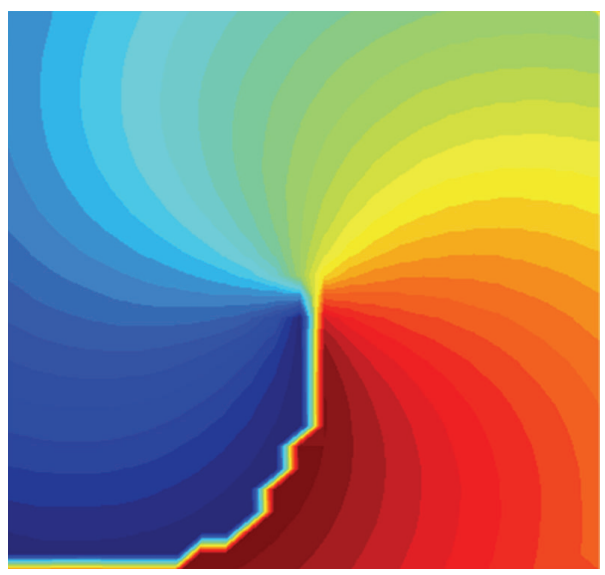

(a)

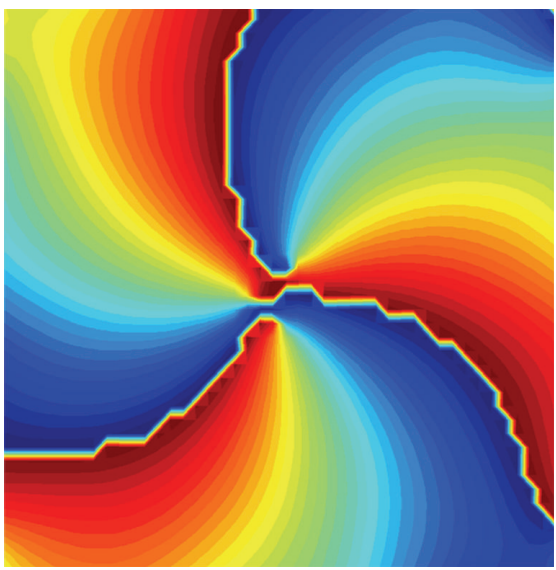

(c)

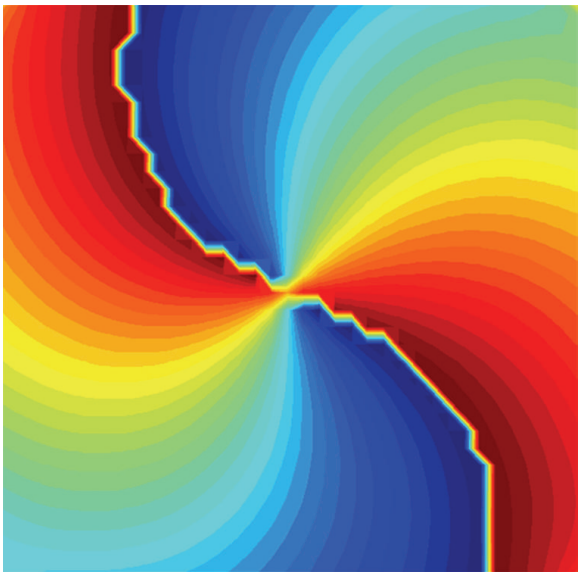

(e)

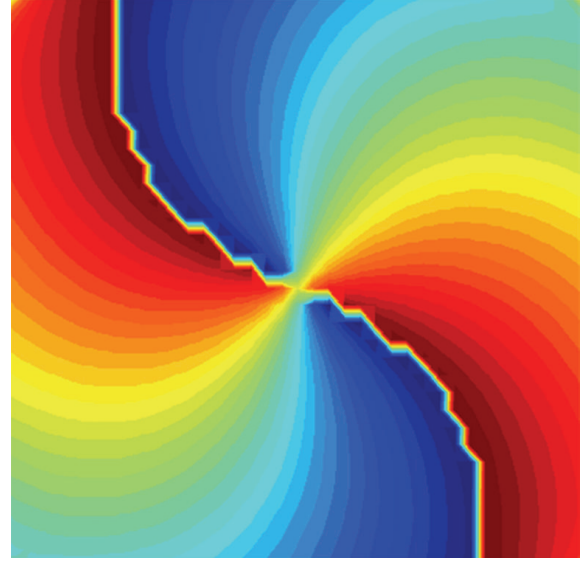

(b)

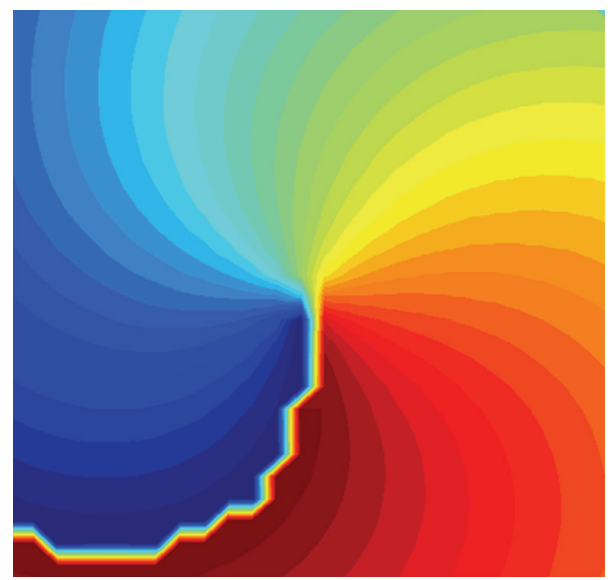

(d)

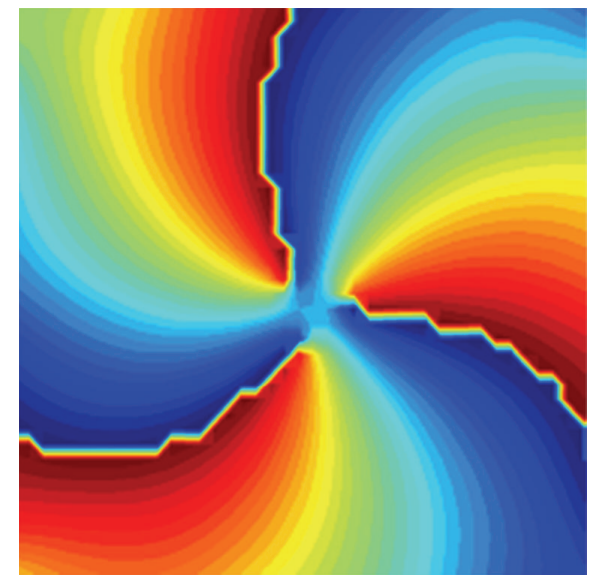

(f)

FIGURE 12: The helical phase front of OAM radio beams. Linear polarization: (a) $\ell=+1$, (b) $\ell=+2$, and (c) $\ell=+3$. Right circular polarizations: (d) $\ell=+1$, (e) $\ell=+2$, and (f) $\ell=+3$.

From (2), we know that the capacity of wireless communication systems can also be enhanced by using OAM beams as information carriers for multiplexing, since each of these orthogonal OAM eigenstates of the total superimposed noninteger OAM beam can be demodulated. So we further investigate the superposition of two concentric orthogonal
OAM states by adopting two coaxial circular antenna arrays with different radii. The number of the outer antenna array is still $N_{\text {out }}=10$, while the inner is $N_{\text {in }}=6$. For the outer array, the phase increment of successive antennas is $2 \pi \ell_{\text {out }} / N_{\text {out }}$, and for the inner the phase increment is $2 \pi \ell_{\text {in }} / N_{\text {in }}$; here $\ell_{\text {out }}$ and $\ell_{\text {in }}$ are the corresponding topological charges. 


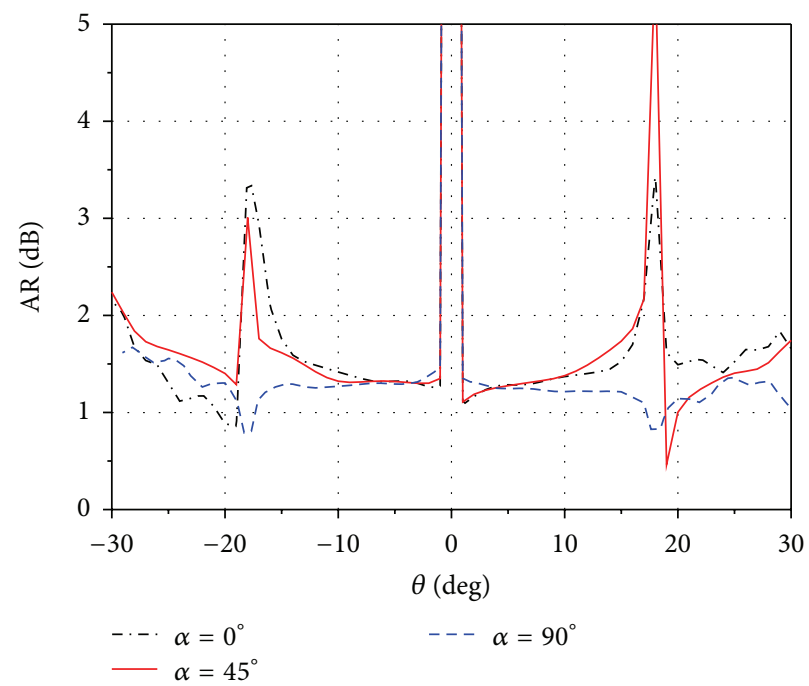

Figure 13: The AR of OAM state $\ell=-1$ for right circular polarization.

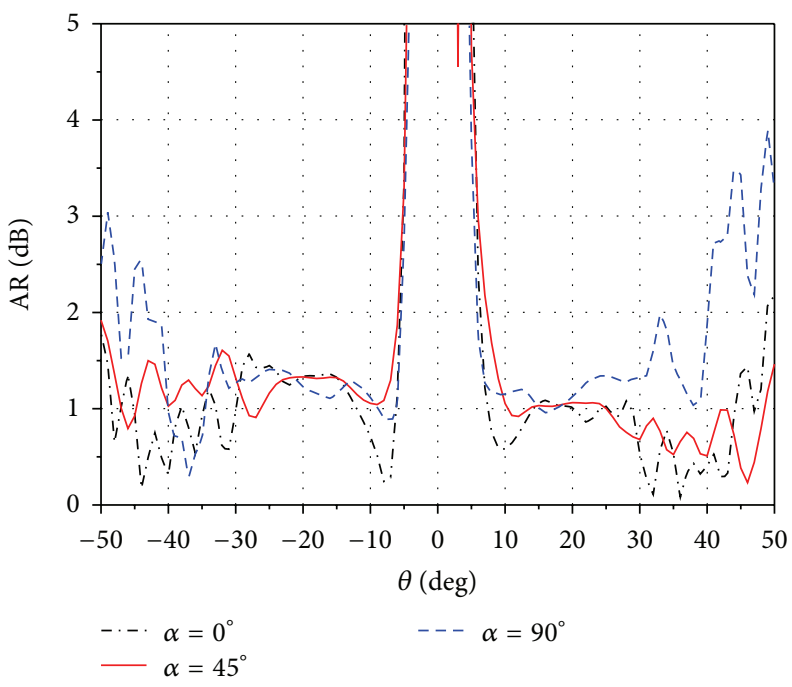

FIgURE 14: The AR of OAM state $\ell=+3$ for left circular polarization.

The superimposed radiation patterns are shown in Figure 15 for four different combinations of $\ell_{\text {out }}$ and $\ell_{\text {in }}$; it is observed that the superimposed patterns are resynthesized and the number of the main beam for the superposition states is $\left|\ell_{\text {out }}-\ell_{\text {in }}\right|$.

3.7. Measurement of OAM States. Since each OAM state comprises a corresponding azimuthal phase term $\exp (i \ell \theta)$, the OAM states can thus be measured through the phase difference of two identical receiving antennas which are set up in an interferometric configuration. The receiving antennas should be oriented along the same direction transverse to the radio beam axis and spaced along the perimeter of a circle perpendicular to the array axis with strong beam intensity, as shown in Figure 16. By distinguishing the number of helical wavefronts from the phase difference of two fixed positions, the OAM state can be finally determined. This measuring method is similar to that shown in literature $[9,12]$.

\section{Conclusion}

In summary, an effective solution of generating OAMcarrying radio beams with three polarizations is presented. It is the first time to adopt horn antenna to provide good OAM states with all three polarizations in the low-frequency radio domain. Through the using of modern digital radio techniques, good OAM-carrying radio waves with three polarizations are obtained and the electric field vectors with different OAM states for the linear as well as the left and right circular polarizations are presented. Simulations show that the results are quite consistent with the traditional optical states. All of the research lays the foundation for new methods 


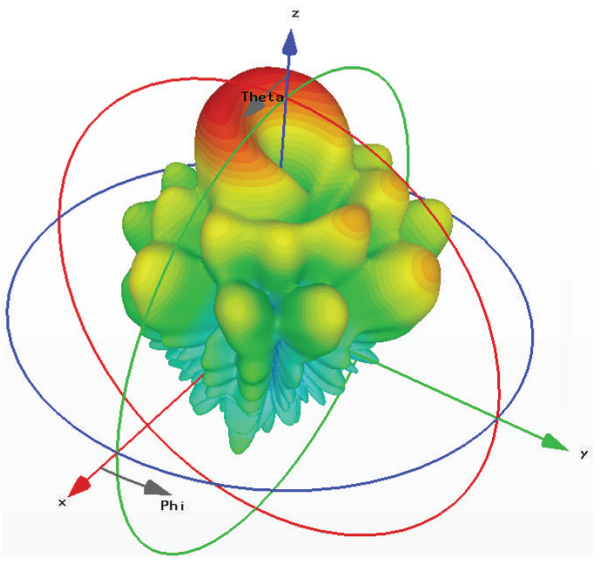

(a)

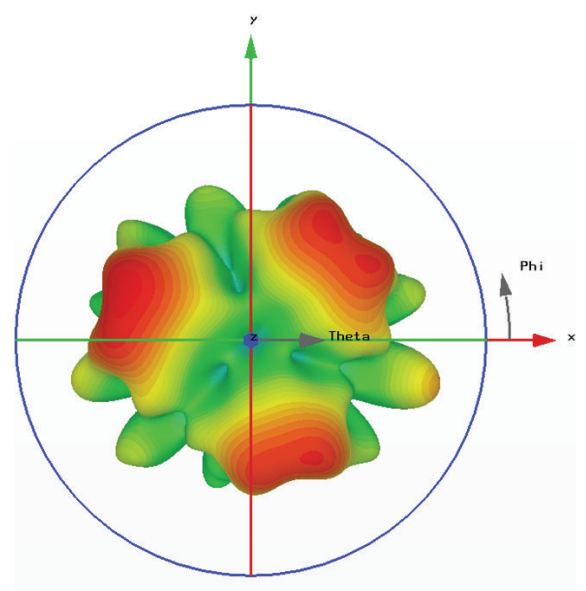

(c)

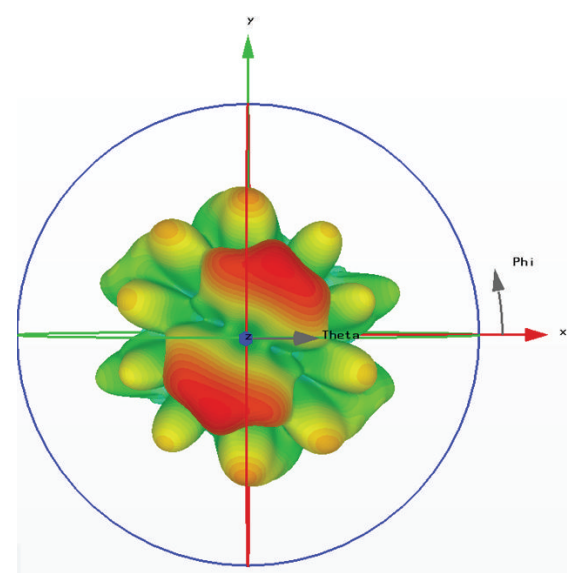

(b)

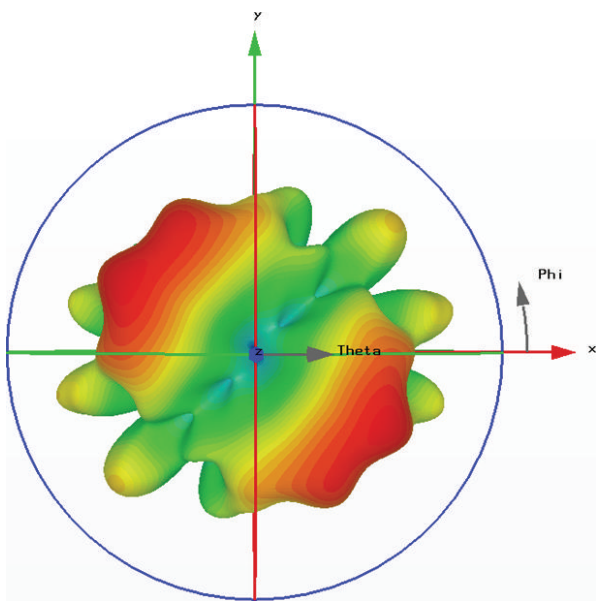

(d)

Figure 15: Radiation patterns of superimposing two different OAM states: (a) $\ell_{\text {out }}=2, \ell_{\text {in }}=1$; (b) $\ell_{\text {out }}=3, \ell_{\text {in }}=1$; (c) $\ell_{\text {out }}=4, \ell_{\text {in }}=1$; (d) $\ell_{\text {out }}=4, \ell_{\text {in }}=2$.

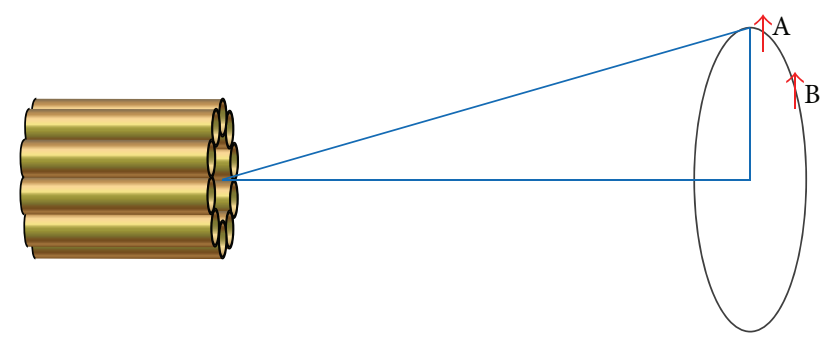

FIGURE 16: Schematic of the OAM reception.

and techniques which can vastly increase the capacity of wireless communication and the spectral efficiency, since information can be encoded as OAM states or, OAM beams associated with polarization diversity can be used as information carriers for multiplexing.

\section{Conflict of Interests}

The authors declare that there is no conflict of interests regarding the publication of this paper.

\section{Acknowledgments}

This work was supported by the National Science and Technology of Major Project under Grant 2011ZX03001-00703, the National Natural Science Foundation under Grant 61201058, the State Quality Inspection Administration of Science and Technology Project under Grant 2013QK127, the Research and Innovation Project of Shanghai Education Commission under Grant 12Z112030001, and the Project of "SMC Excellent Young Faculty."

\section{References}

[1] J. D. Kraus and R. J. Marhefka, Antennas: For All Applications, McGraw-Hill International, 2002.

[2] D. Dardari and V. Tralli, "High-speed indoor wireless communications at $60 \mathrm{GHz}$ with coded OFDM," IEEE Transactions on Communications, vol. 47, no. 11, pp. 1709-1721, 1999.

[3] D. K. Cheng, Field and Wave Electromagnetics, Addison-Wesley, Reading, Mass, USA, 2nd edition, 1983.

[4] R. A. Beth, "Mechanical detection and measurement of the angular momentum of light," Physical Review, vol. 50, no. 2, pp. 115-125, 1936. 
[5] L. Allen, M. W. Beijersbergen, R. J. C. Spreeuw, and J. P. Woerdman, "Orbital angular momentum of light and the transformation of Laguerre-Gaussian laser modes," Physical Review A, vol. 45, no. 11, pp. 8185-8189, 1992.

[6] J. H. Shapiro, S. Guha, and B. I. Erkmen, "Ultimate channel capacity of free-space optical communications," Journal of Optical Networking, vol. 4, pp. 501-516, 2005.

[7] J. Wang, J.-Y. Yang, I. M. Fazal et al., “Terabit free-space data transmission employing orbital angular momentum multiplexing," Nature Photonics, vol. 6, no. 7, pp. 488-496, 2012.

[8] B. Thidé, H. Then, J. Sjöholm et al., "Utilization of photon orbital angular momentum in the low-frequency radio domain," Physical Review Letters, vol. 99, no. 8, Article ID 087701, 2007.

[9] F. Tamburini, E. Mari, B. Thidé, C. Barbieri, and F. Romanato, "Experimental verification of photon angular momentum and vorticity with radio techniques," Applied Physics Letters, vol. 99, no. 20, Article ID 204102, 2011.

[10] F. Tamburini, E. Mari, A. Sponselli, B. Thidé, A. Bianchini, and F. Romanato, "Encoding many channels on the same frequency through radio vorticity: first experimental test," New Journal of Physics, vol. 14, Article ID 033001, 2012.

[11] S. M. Mohammadi, L. K. S. Daldorff, J. E. S. Bergman et al., "Orbital angular momentum in radio-a system study," IEEE Transactions on Antennas and Propagation, vol. 58, no. 2, pp. 565-572, 2010.

[12] C. Deng, W. Chen, Z. Zhang, Y. Li, and Z. Feng, "Generation of OAM radio waves using circular vivaldi antenna array," International Journal of Antennas and Propagation, vol. 2013, Article ID 847859, 7 pages, 2013.

[13] M. H. Chen and G. N. Tsandoulas, "A wideband squarewaveguide array polarizer," IEEE Transactions on Antennas and Propagation, vol. 21, no. 5, pp. 389-391, 1973.

[14] M. V. Berry, "Optical vortices evolving from helicoidal integer and fractional phase steps," Journal of Optics A: Pure and Applied Optics, vol. 6, no. 2, pp. 259-268, 2004. 

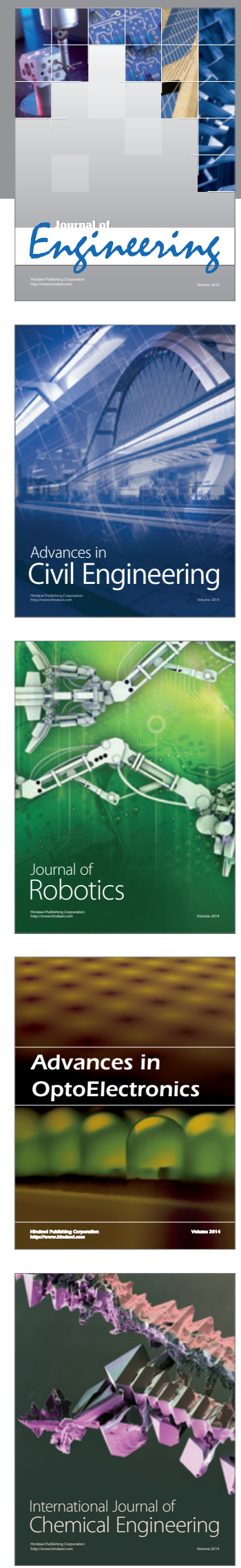

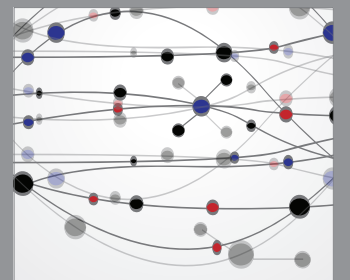

The Scientific World Journal
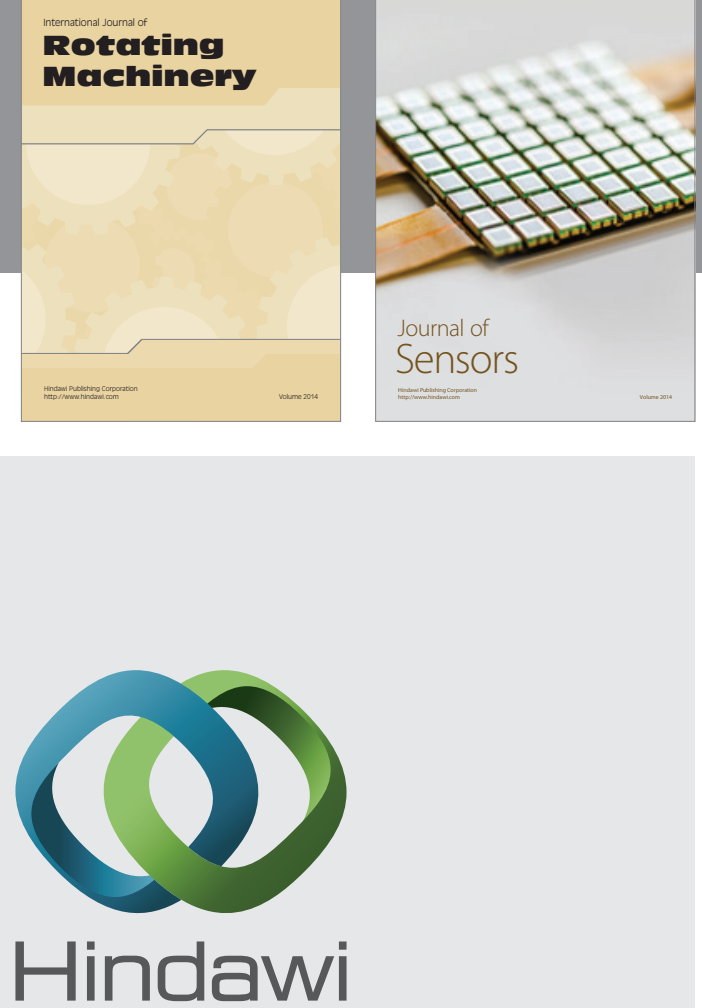

Submit your manuscripts at http://www.hindawi.com
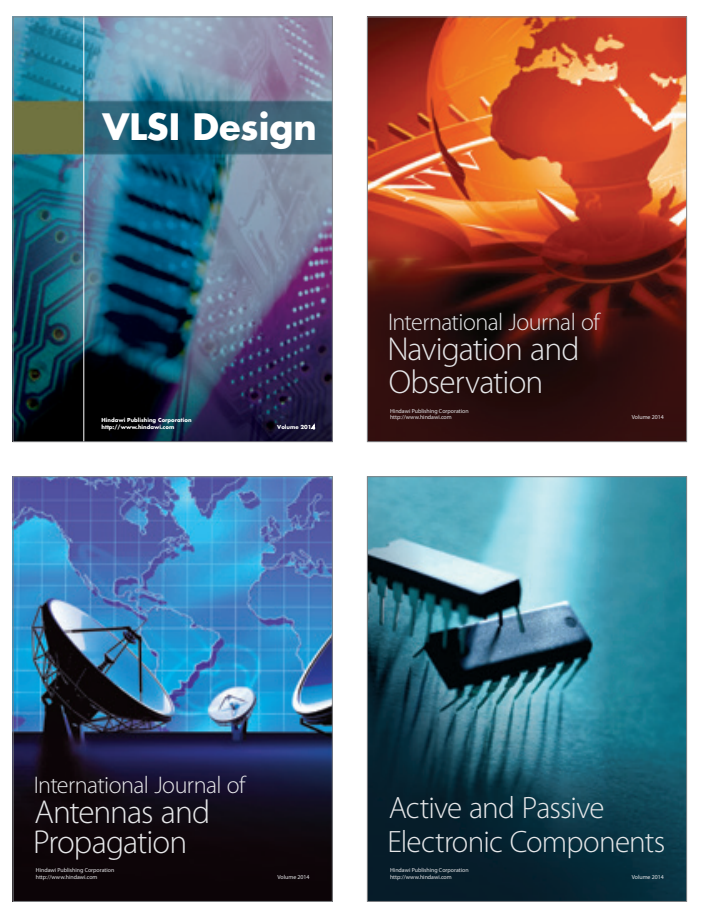
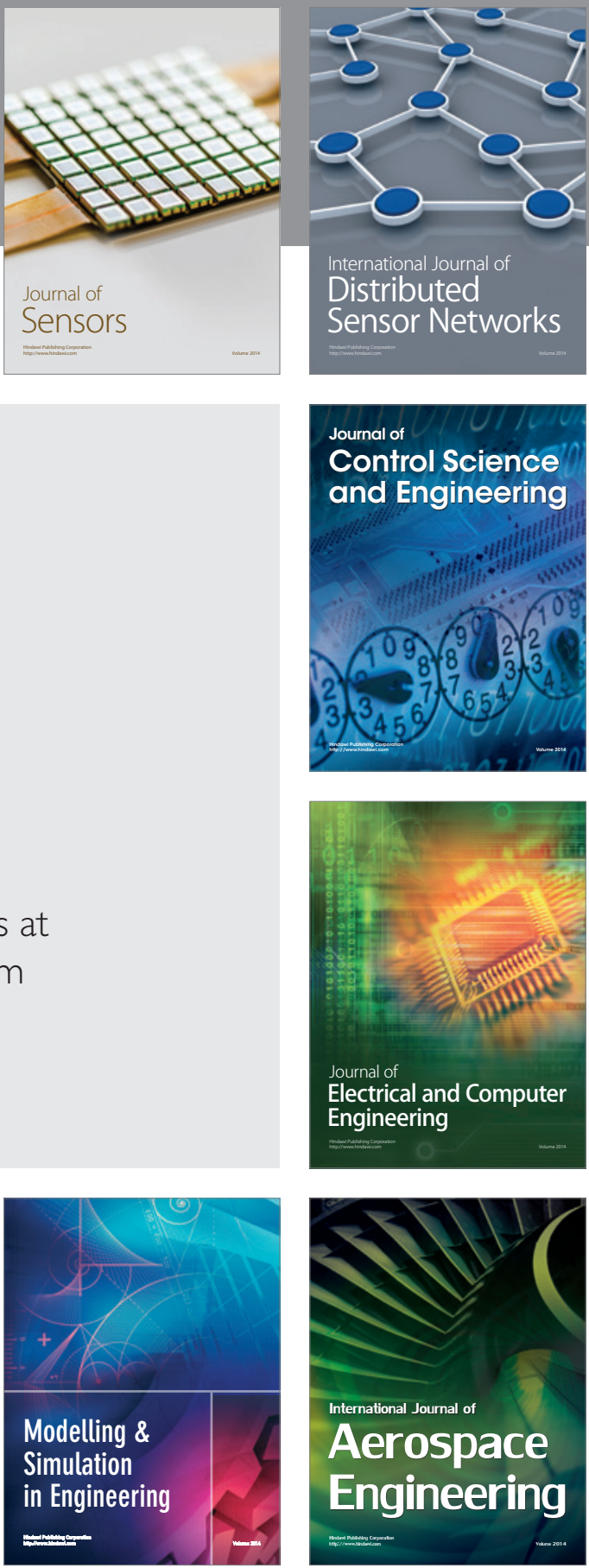

Journal of

Control Science

and Engineering
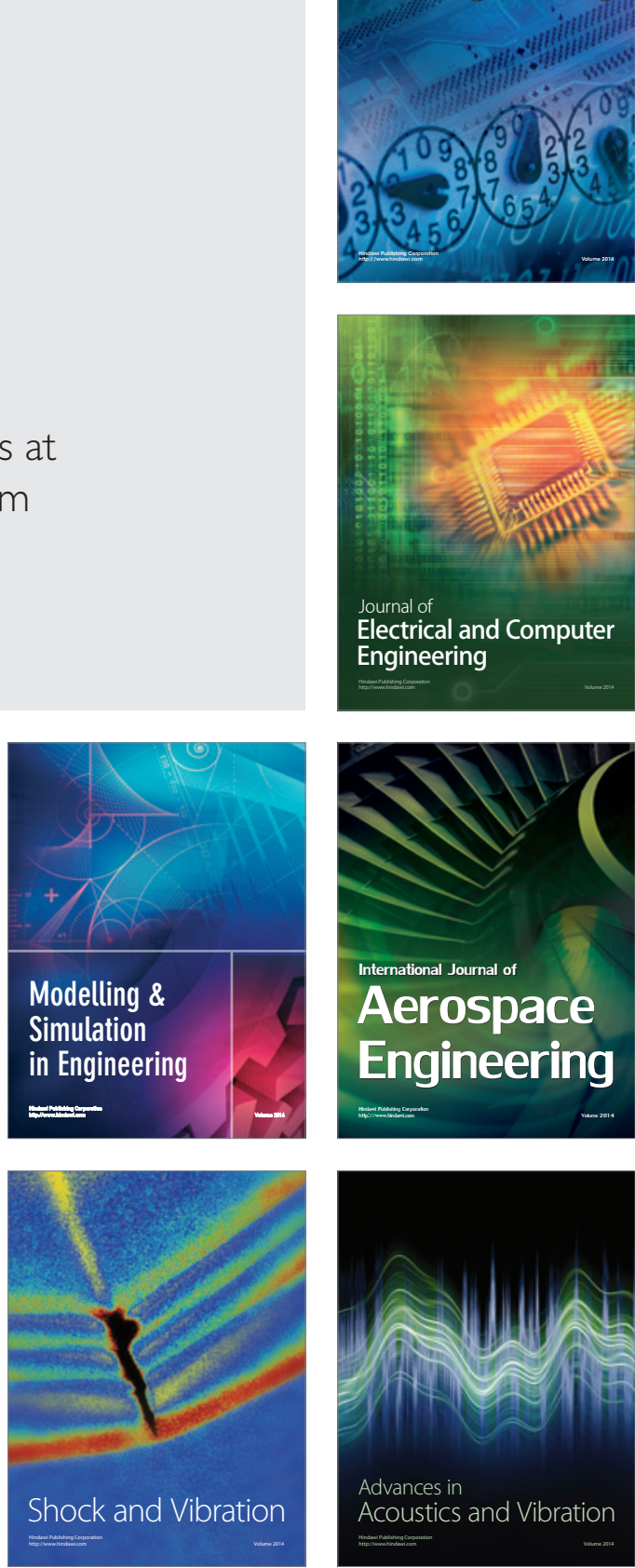